\title{
Conformational landscape of
}

\section{meso-(1,3-dithian-2-yl)-porphyrins}

\author{
Supplementary Information
}

Philipp Wacker, ${ }^{\dagger}$ Katja Dahms, ${ }^{\ddagger}$ Mathias O. Senge ${ }^{\ddagger}$ and Erich Kleinpeter*, ${ }^{\dagger}$

Institut für Chemie, Universität Potsdam, Karl-Liebknecht-Strasse 24-25, D-14476 Golm, Germany and

School of Chemistry, SFI Tetrapyrrole Laboratory, University College Dublin, Trinity College, Dublin 2, Ireland

kp@chem.uni-potsdam.de

${ }^{\dagger}$ Universität Potsdam

${ }^{\ddagger}$ Trinity College Dublin

Figure S1. Assignment of the ${ }^{1} \mathrm{H}$ NMR spectra for the aromatic region for porphyrin $\mathbf{1}$ at $298 \mathrm{~K}$ (top) and

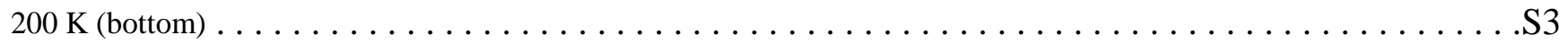

Figure S2. Assignment of the ${ }^{1} \mathrm{H}$ NMR spectra for the aromatic region for porphyrin 2 at $298 \mathrm{~K}$ (top) and

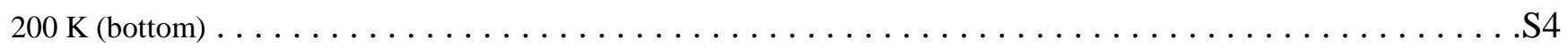

Figure S3. Assignment of the ${ }^{1} \mathrm{H}$ NMR spectra for the aromatic region for porphyrin 3 at $298 \mathrm{~K}$ (top) and $200 \mathrm{~K}$ (bottom)

Figure S4. Assignment of the ${ }^{1} \mathrm{H}$ NMR spectra for the aromatic region for porphyrin 4 at $298 \mathrm{~K}$ (top) and $200 \mathrm{~K}$ (bottom) S6 


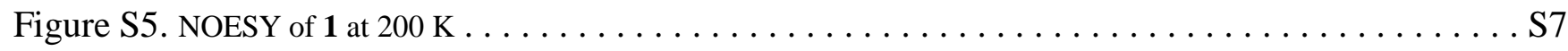

Table S1. Energies of optimized structures and transition states; $\mathrm{E}($ total $)=\mathrm{HF}+$ zero-point vibrational

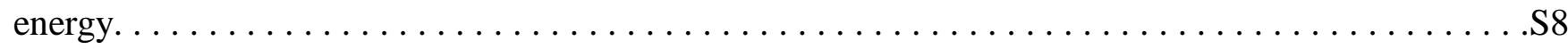

Listing $\mathrm{S} 1$ : Coordinates of calculated structures. $\ldots \ldots \ldots \ldots \ldots \ldots \ldots \ldots \ldots \ldots \ldots \ldots \ldots$ 


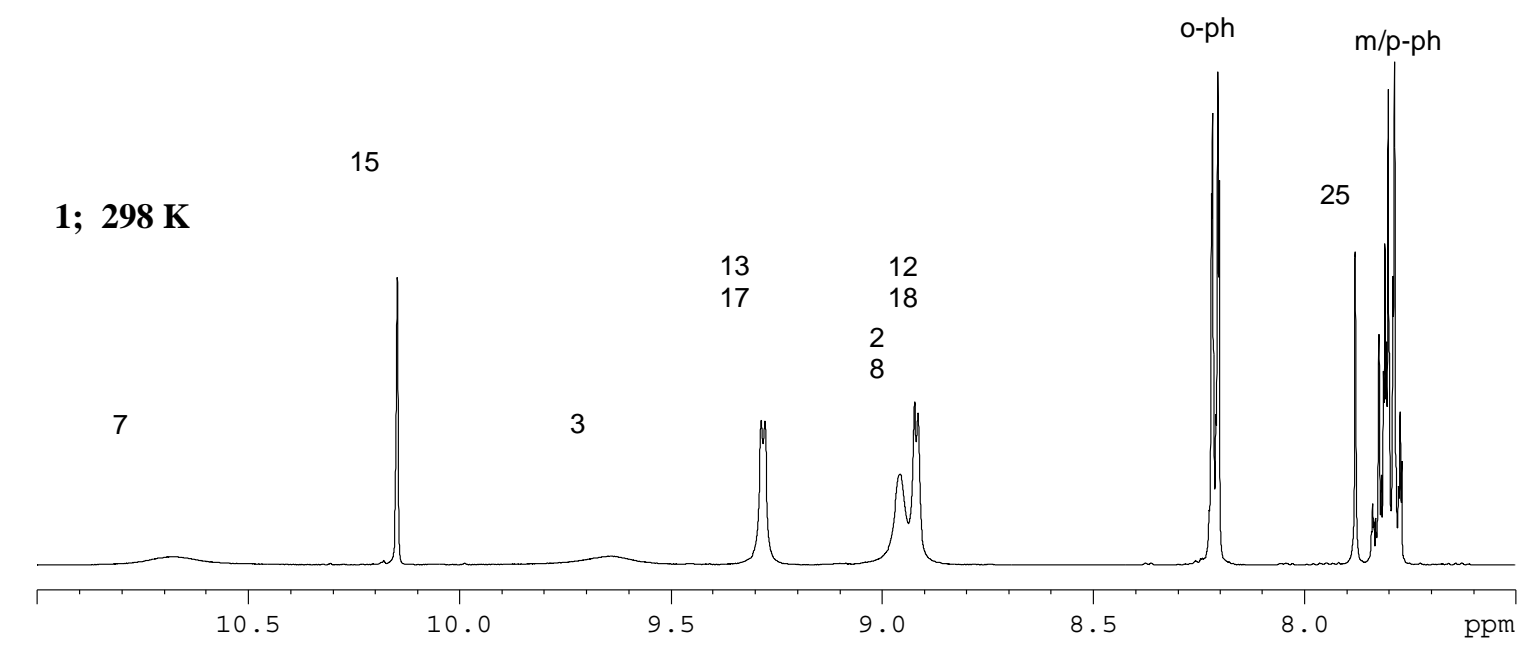

$1 ; 200 \mathrm{~K}$

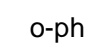

m/p-ph

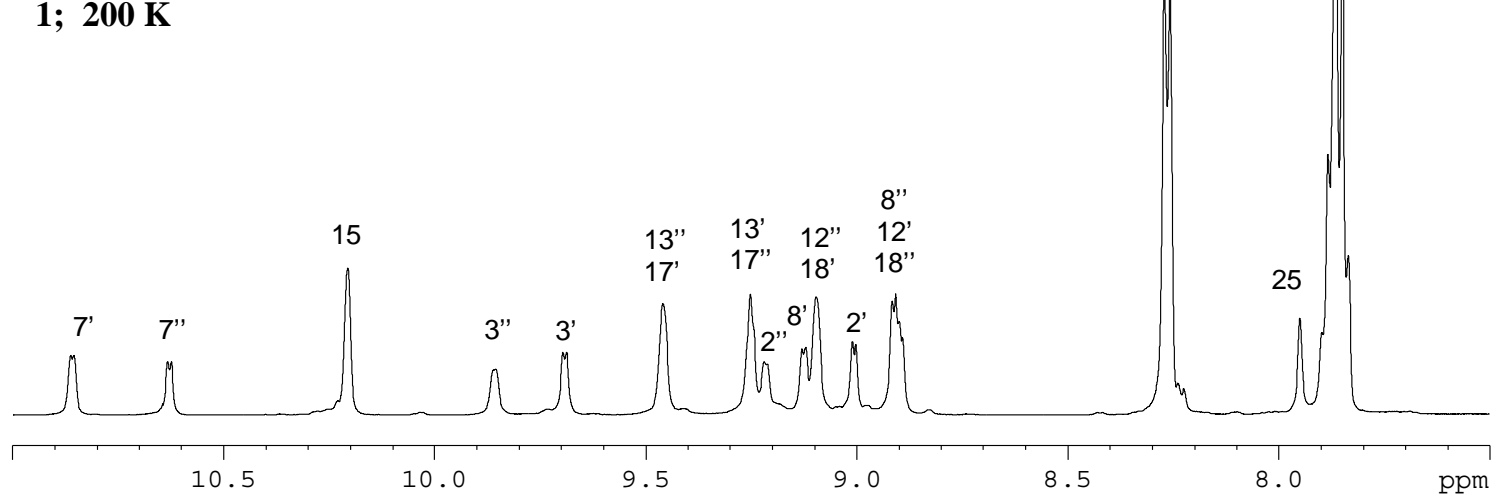

Figure S1: Assignment of the ${ }^{1} \mathrm{H}$ NMR spectra for the aromatic region for porphyrin $\mathbf{1}$ at $298 \mathrm{~K}$ (top) and $200 \mathrm{~K}$ (bottom) in $\mathrm{CD}_{2} \mathrm{Cl}_{2}$ at $500 \mathrm{MHz}$. Signal intensities were adapted for illustrative purposes. 


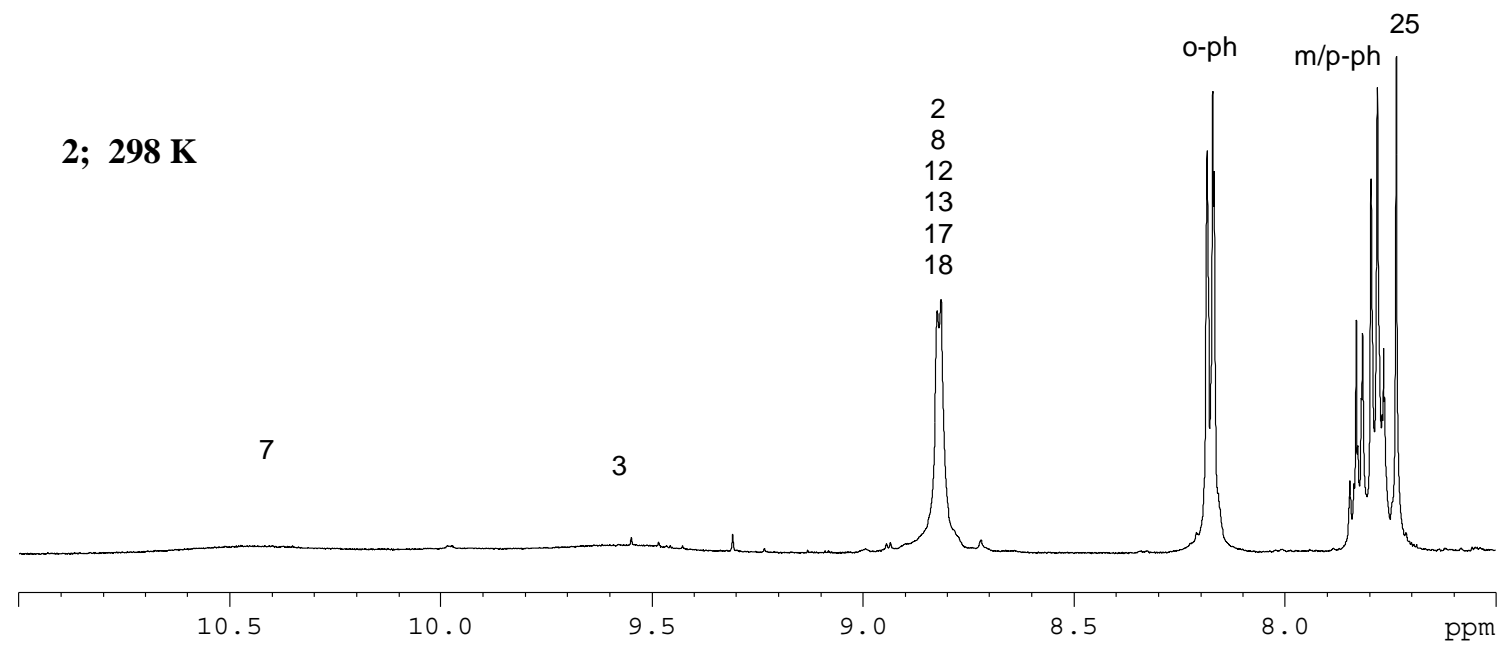

$2 ; 200 \mathrm{~K}$

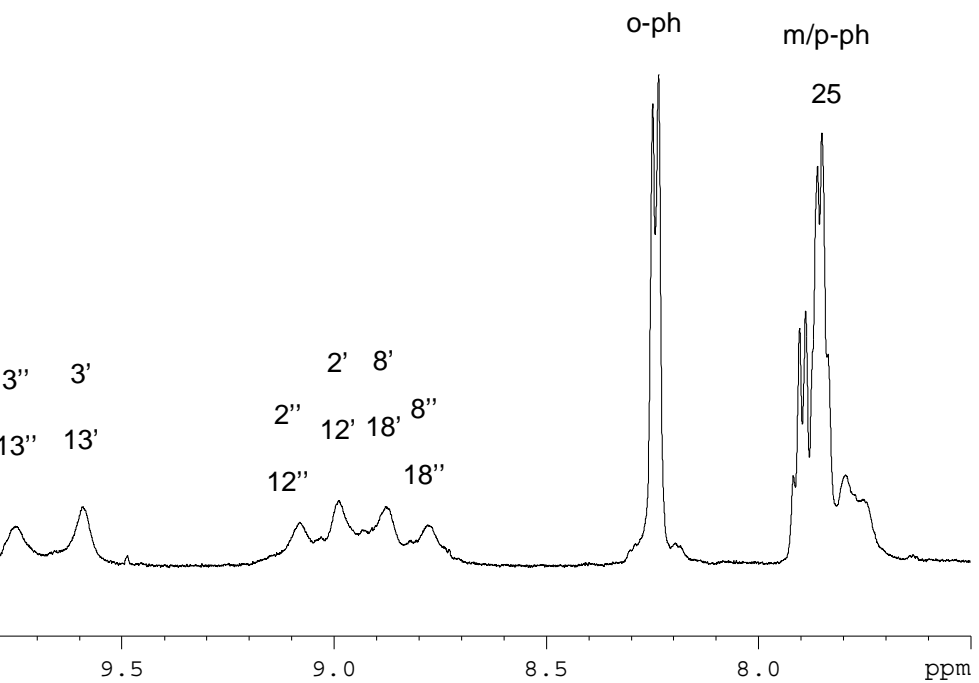

Figure S2: Assignment of the ${ }^{1} \mathrm{H}$ NMR spectra for the aromatic region for porphyrin 2 at $298 \mathrm{~K}$ (top) and $200 \mathrm{~K}$ (bottom) in $\mathrm{CD}_{2} \mathrm{Cl}_{2}$ at $500 \mathrm{MHz}$. Signal intensities were adapted for illustrative purposes. 


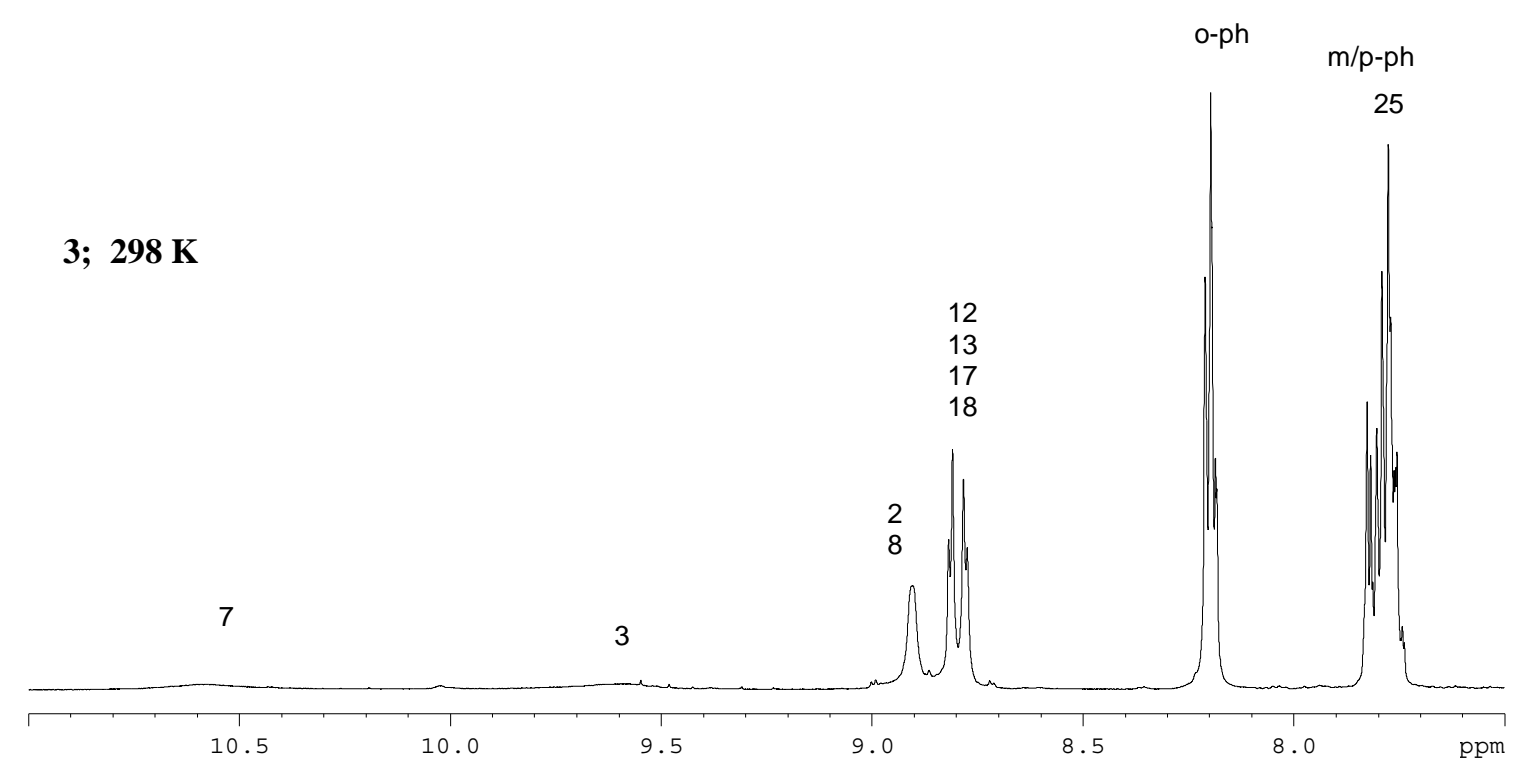

3; $200 \mathrm{~K}$

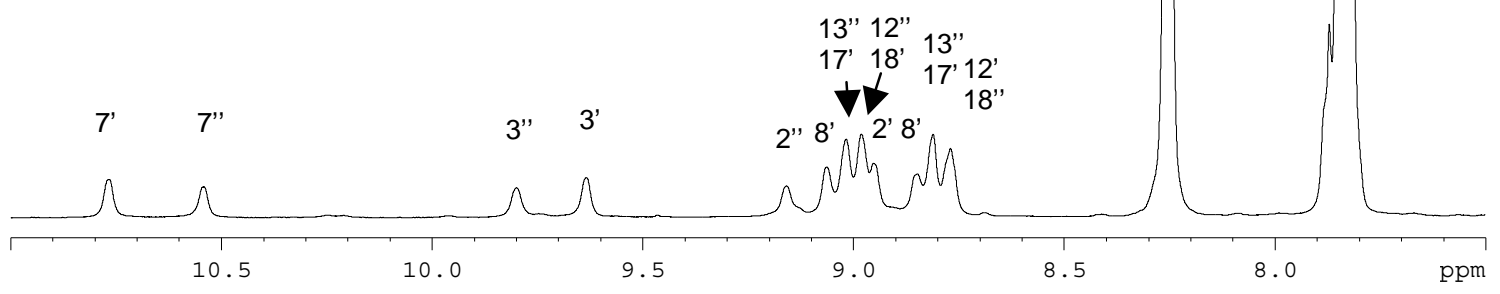

Figure S3: Assignment of the ${ }^{1} \mathrm{H}$ NMR spectra for the aromatic region for porphyrin 3 at $298 \mathrm{~K}$ (top) and $200 \mathrm{~K}$ (bottom) in $\mathrm{CD}_{2} \mathrm{Cl}_{2}$ at $500 \mathrm{MHz}$. Signal intensities were adapted for illustrative purposes. 

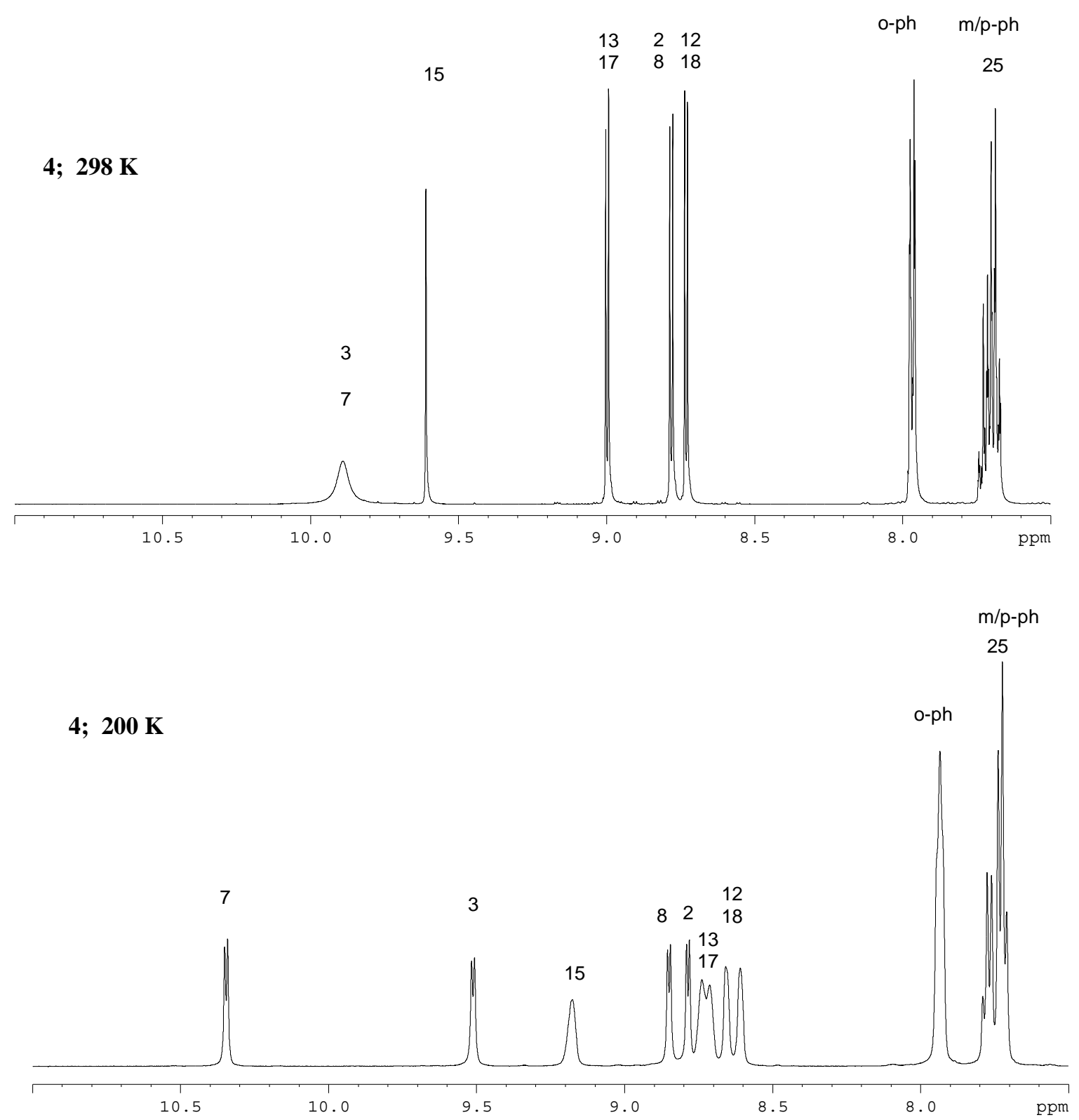

Figure S4: Assignment of the ${ }^{1} \mathrm{H}$ NMR spectra for the aromatic region for porphyrin 4 at $298 \mathrm{~K}$ (top) and $200 \mathrm{~K}$ (bottom) in $\mathrm{CD}_{2} \mathrm{Cl}_{2}$ at $500 \mathrm{MHz}$. Signal intensities were adapted for illustrative purposes. 


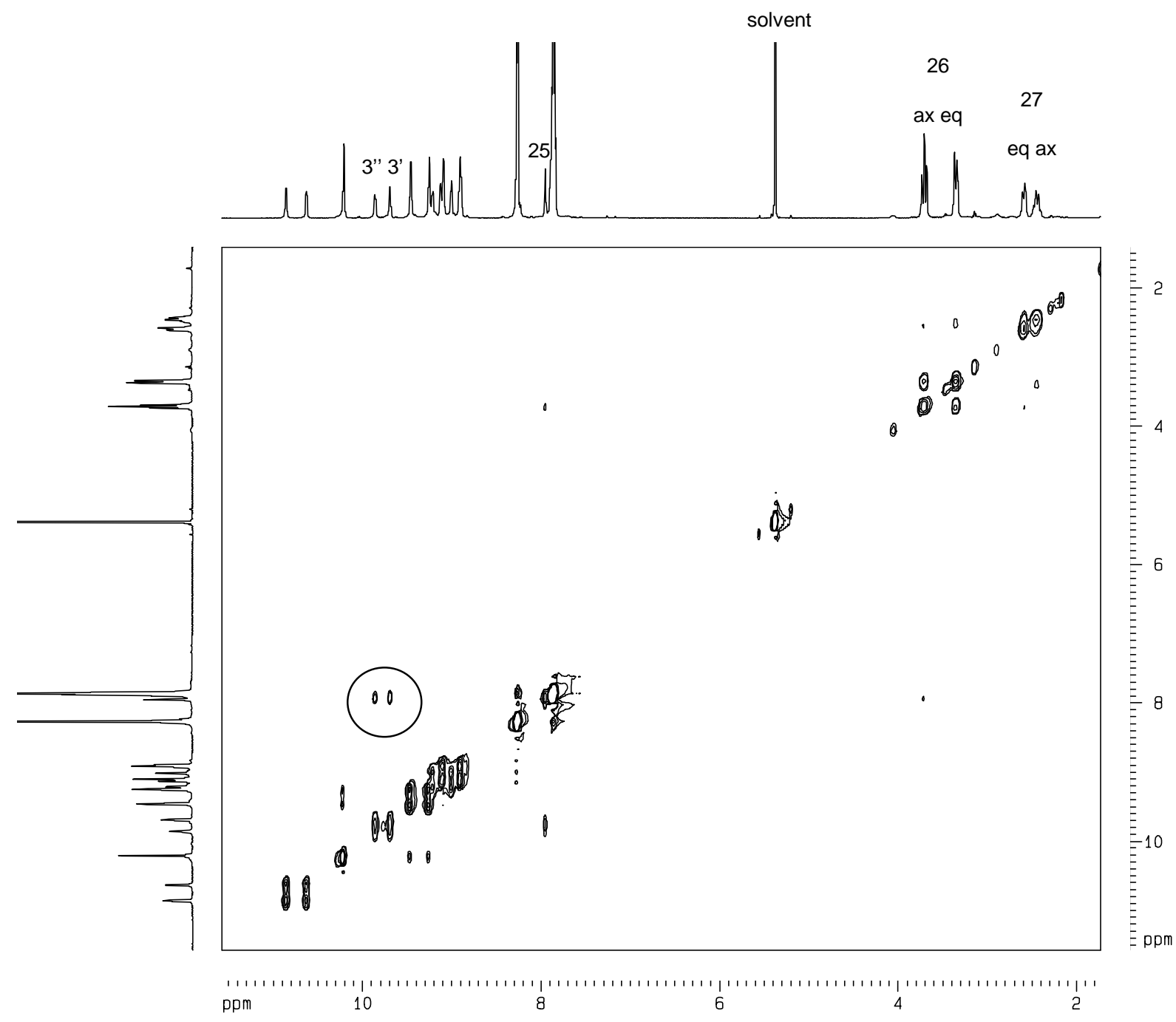

Figure S5: NOESY of 1 at $200 \mathrm{~K}$ in $\mathrm{CD}_{2} \mathrm{Cl}_{2}$. The cross peaks $\mathrm{H} 25-\mathrm{H}^{\text {/" }}$ are marked. With this information at hand, the orientation of the dithianyl moiety is clarified. 
Table S1. Energies of optimized structures and transition states; E(total) $=\mathrm{HF}+$ zero-point vibrational energy

\begin{tabular}{cccc}
\hline $\begin{array}{c}\text { Structur } \\
\text { e }\end{array}$ & B3LYP/6-31G & B HLYP/6-31G & E HF \\
\hline & & & B3LYP/6-31G** \\
\hline 1A & -2403.6009083 & -2403.041777 & -2404.1096517 \\
1B & --- & --- & -2404.1089213 \\
1TS & -2403.57545 & -2403.017292 & --- \\
2A & -3355.9182731 & -3355.263155 & -3356.5290052 \\
2B & --- & --- & -3356.5276082 \\
2C & --- & --- & -3356.5283215 \\
2TS & -3355.8949058 & -3355.240760 & --- \\
3A & -2634.5991009 & -2633.958431 & -2635.1657459 \\
3B & --- & ---- & -2635.1651443 \\
3TS & -2634.5753172 & -2633.935486 & -3911.1787532 \\
$\mathbf{4}$ & -3910.672765 & -3910.134325 & -- \\
$\mathbf{4 T S}$ & -3910.65496 & -3910.117268 & \\
\hline & & & \\
\hline
\end{tabular}


Listing S1: Coordinates of calculated structures

1A (B3LYP/6-31G**)

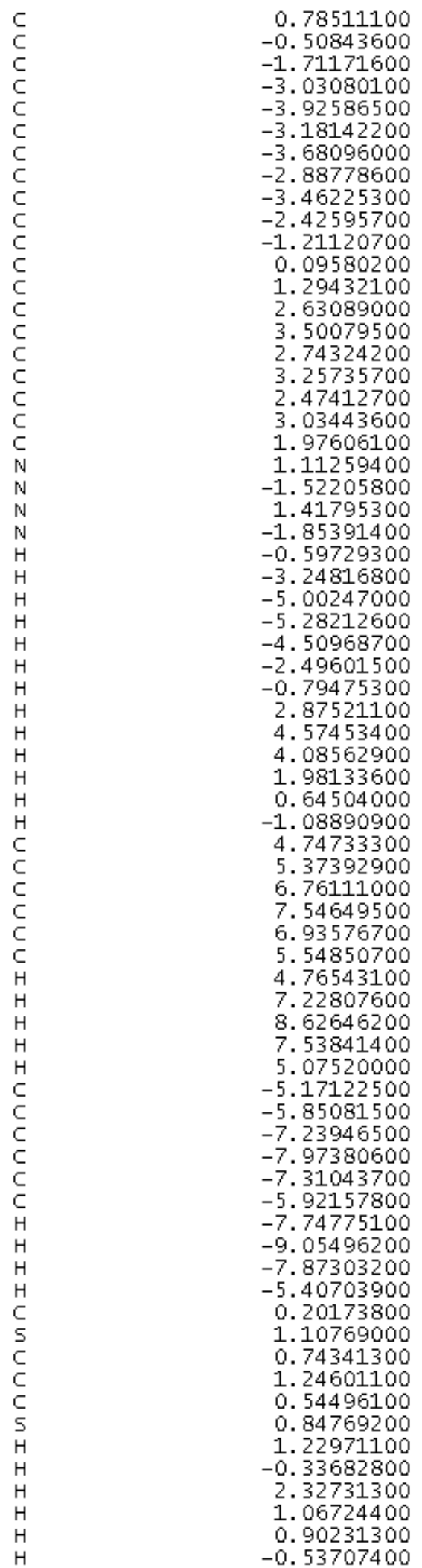

$-4.16434100$

$-4.68561000$

$-3.98574400$

$-4.53344000$

$-3.49660500$

$-2.27625600$

$-0.96568000$

0.20118800

1. 52866000

2.39723600

1. 59891800

2.15188000

1.41347400

1.93509300

0.88168000

$-0.33038300$

$-1.63714900$

$-2.81023200$

$-4.15766500$

$-5.00024400$

$-2.84404700$

0.27104600

0.04305400

$-2.62577600$

$-5.76377900$

$-5.58737900$

$-3.55340600$

0.17866100

1. 75286800

3.46533400

4. 08193700

2. 98173500

0.92528800

$-4.40252500$

$-6.07834100$

$-0.60860800$

$-1.96262300$

$-1.78133700$

$-2.37653200$

$-2.51715500$

$-2.06534300$

$-1.47191000$

$-1.33077700$

$-2.72225900$

$-2.97588000$

$-2.17509100$

$-1.12227700$

$-0.87721300$

$-0.81986000$

$-0.19711700$

$-0.06725000$

$-0.55780400$

$-1.17936800$

$-1.31037700$

0.41351000

$-0.45616600$

$-1.55875600$

$-1.78578000$

3. 66721900

4.25346600

6.04754600

6.68190500

6.16035800

4. 38441700

6. 50874200

6. 20381500

6.53417900

7. 76472800

6. 69174400

6. 32960600
$-0.18036900$

$-0.23666100$

$-0.19269400$

$-0.27291100$

$-0.20439500$

$-0.07330700$

0.01635900

0.07040200

0. 24311700

0.23084600

0.06236800

0.01928000

0.03261500

0.13811400

0.16075000

0.05074600

0.02094500

$-0.05577100$

$-0.17072100$

$-0.24573000$

$-0.06808000$

$-0.01687900$

$-0.01256300$

$-0.07282300$

$-0.32938700$

$-0.37844900$

$-0.24900900$

$-1.86004400$

0.37719000

0.37284500

$-0.14606700$

0.21166900

0.25461500

$-0.20238300$

$-0.34354400$

$-0.08969800$

$-0.00932800$

0.06701800

1.17344400

1. 21728200

0.15547100

$-0.95037400$

$-0.99406900$

2.00356200

2. 08411000

0.18950600

$-1.78370800$

$-1.85966300$

0.04418400

$-1.01499100$

$-0.99326400$

0.08780700

1.14687800

1.12461400

$-1.82416800$

0.10489000

1.99507100

1. 95424700

$-0.02901800$

$-1.53838700$

$-1.37322500$

$-0.07292400$

1.18469300

1.55410000

$-2.23759900$

$-1.48611800$

0.01697600

$-0.13358300$

2. 07136900

1. 11865600 
1B (B3LYP/6-31G**)

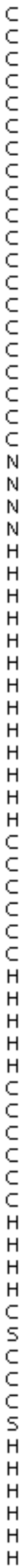

\begin{tabular}{|c|c|c|}
\hline 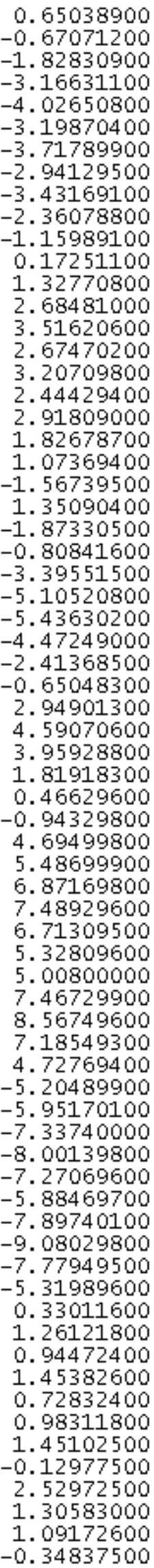 & 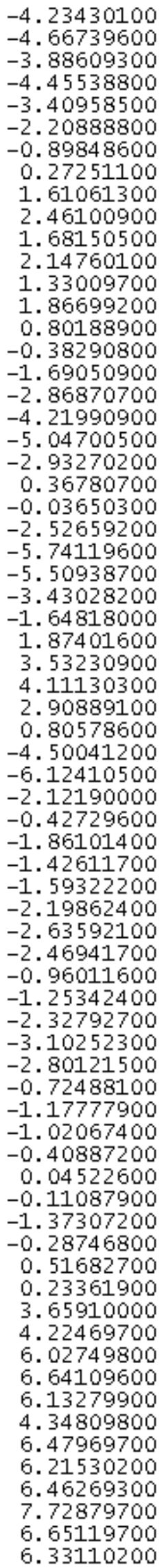 & 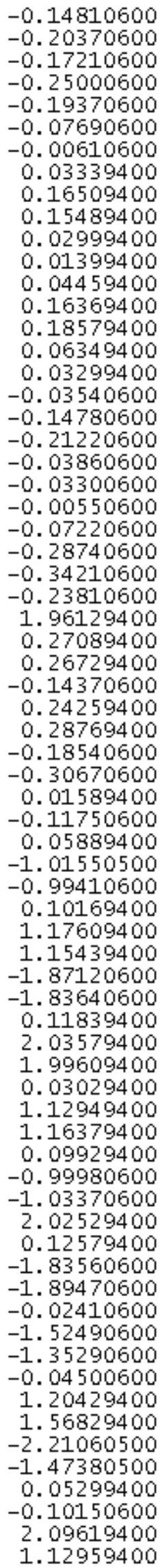 \\
\hline
\end{tabular}

$-0.14810600$

$-0.25000600$

0.00610600

03339400

.15489400

01399400

.04459400

0.06349400

03540600

$-0.14780600$

21220600

$-0.03300600$

$-0.07220600$

$-0.28740600$

0.23810600

1.96129400

0.27089400

0.14370600

0.24259400

$-18540600$

$-0.30670600$

0.05889400

. 99410600

0.10169400

.17609400

$-1.87120600$

$-1.83640600$

.11839400

1. 99609400

0.03029400

.12949400

- 09929400

$-0.99980600$

3370600

$-1.83560600$

$-1.89470600$

$-0.02410600$

$-1.52490600$

$-1.35290600$

04500600

1. 56829400

$-2.21060500$

380500

1.12959400 
1A (B3LYP/6-31G); no imaginary frequency

$-4.19843500$ $-4.70620900$ $-3.99409300$ $-4.53452900$ $-3.48866700$ $-2.26453400$ $-0.95137000$ 0.20972700

1. 55229900 2. 41556600 1. 61198300 2.15208700 1.40201600 1. 91548700 0.85386700 $-0.36077100$ $-1.66968600$ $-2.83759300$ $-4.19831800$ $-5.03724300$ $-2.86511100$ 0.27109500 0.02082100 $-2.62168600$ $-5.78442200$ $-5.58641800$ $-3.54425900$ $-1.76347800$ 1. 78985900 3.48475800 4.10618500 2. 95849300 0.89596900 $-4.45482800$ $-6.11472100$ $-0.62894100$ $-1.95931700$ $-1.82259100$ $-1.36475700$ -1. 51490300 $-2.12430300$ $-2.58348100$ $-2.43453300$ $-0.90119000$ $-1.16111600$ $-2.24033700$ $-3.05280800$ $-2.78394200$ $-0.79534700$ $-1.28395200$ $-1.14217500$ $-0.51168900$ $-0.02276900$ $-0.16304700$ $-1.51851900$ $-0.40219100$ 0.46307900 0.20941200 3.65858500 4. 28537000 6.13636000 6.73658300 6.22439700 4. 38898200 6.60653300 6.26633900 6.56675500 7.82664300 6.74812700 6.36511600
$-0.15489000$

$-0.20157700$

$-0.16319100$

$-0.23351400$

$-0.17704500$

$-0.06269700$

0.01204800

0.05932900

0.20939900

0.20129000

0.05732400

0.02288800

0.03007400

0.11905400

0.13588500

0.04067500

0.01379600

$-0.04923400$

$-0.15525700$

$-0.21683700$

$-0.05583000$

$-0.01323200$

$-0.01154500$

$-0.06067400$

$-0.28174000$

$-0.32429000$

$-0.21952700$

1. 94237200

0.32651000

0.32489000

$-0.10168600$

0.18230700

0.21645200

$-0.19086400$

$-0.30474800$

$-0.06745000$

$-0.01219900$

0.04977600

$-1.01525600$

$-0.98090000$

0.11867500

1.18383800

1.14984700

$-1.87360700$

$-1.81366200$

0.14516300

2.04259800

1. 98026700

0.03170500

1. 11082600

1.12582400

0.06148800

-1. 01779900

$-1.03288900$

1. 96958400

0.07312900

$-1.84953300$

$-1.87422400$

$-0.01217300$

$-1.56908300$

$-1.34847500$

$-0.04560200$

1. 22226000

1.60696400

$-2.21871100$

$-1.42928800$

0.02402100

$-0.09138900$

2.10845800

1.16804300 
$1 T S(B 3 L Y P / 6-31 G)$; one imaginary frequency

$-1.06620000$

0.21464100

1.42499800

2.72309100

3.64402600

2. 91369400

3. 50990900

2.83170300

3.42245200

2.45743300

1. 21107400

$-0.06938600$

$-1.25962000$

$-2.48511900$

$-3.36139300$

$-2.70410200$

$-3.34941200$

$-2.71693900$

$-3.32036700$

$-2.31777500$

$-1.35901500$

1. 48746500

$-1.39780500$

1. 56881700

0.26536300

2. 88562300

4. 70558400

5. 58243200

4.45506800

2. 58397300

$-0.15154500$

$-2.62859900$

$-4.34663300$

$-4.37817500$

$-2.42393200$

$-0.67221900$

0.81236100

$-4.81483400$

$-5.29669100$

$-6.66549200$

$-7.57861400$

$-7.11325700$

$-5.74446200$

$-4.59080500$

$-7.01687500$

$-8.64106200$

$-7.81486300$

$-5.38742900$

4. 98202100

5.92739000

7.29796300

7.74622000

6.81543600

5.44469300

8.01347400

8.80965300

7.15444500

4.72405200

$-0.17866300$

$-1.82503700$

$-1.66499600$

$-0.47261700$

0.87324400

1. 26695600

$-2.61216300$

$-1.63562800$

$-0.51917300$

$-0.54522300$

1. 70442400

0.94064200
$-3.90055800$

$-4.38856800$

$-3.77490400$

$-4.43663200$

$-3.57477500$

$-2.36156500$

$-1.20363400$

$-0.00156200$

1.23771700

2. 21164100

1. 61308300

2. 20660800

1.43172400

1. 91804900

0.87911700

$-0.24347600$

$-1.45488300$

$-2.56321000$

$-3.76757700$

$-4.57390000$

$-2.68554400$

0.26697700

0.10088200

$-2.50924000$

$-5.38089300$

$-5.43272100$

$-3.72890400$

$-1.25854400$

1.35781100

3. 24283700

4. 25260600

2. 89642200

0.86206400

$-3.97231500$

$-5.54929700$

$-1.97459300$

$-0.42072500$

$-1.58495100$

$-2.59236600$

$-2.71878500$

$-1.84360000$

$-0.83998600$

$-0.71072600$

$-3.26573800$

$-3.49565500$

$-1.94262300$

$-0.16082700$

0.06242600

$-1.22672600$

$-1.25947800$

$-1.28270400$

$-1.27183800$

$-1.23739800$

$-1.21519300$

$-1.30402400$

$-1.28966600$

$-1.23266700$

$-1.19760200$

3.73561600

4. 25751400

6.14686700

6.73700000

6.32274400

4. 47122600

6.50482400

6.40974900

6.45386200

7.83520900

6.80180000

6. 58881100
$-1.21816100$

$-1.47690100$

$-1.15739500$

$-1.26207600$

$-0.74830300$

$-0.36383100$

0.18139500

0.43630400

0.86546800

0.83585100

0.41125900

0.35382400

0.49438200

1.14245700

1.16544400

0.50235900

0.18889700

$-0.41089300$

$-0.91779600$

$-1.41365700$

$-0.62817000$

0.21386600

0.16612500

$-0.61917700$

$-1.91416800$

$-1.64957000$

$-0.63687700$

$-1.59777700$

1.14806300

1.10946500

1.19738500

1. 56674700

1. 60311100

$-0.90971800$

$-1.86434500$

$-0.39984800$

$-0.10195300$

0.46620800

1. 32528800

1. 58284200

0.98290300

0.12510100

$-0.13069900$

1.80062500

2.25480100

1.18180900

$-0.34913300$

$-0.80266500$

0.47346500

$-0.56878900$

$-0.28853500$

1. 03755100

2. 08233800

1. 80266100

$-1.10469600$

1.25424600

3.11365700

2. 61414000

0.23139900

$-0.62488800$

$-0.48562800$

$-1.23614500$

$-0.64447100$

$-0.81723700$

$-0.89788600$

0.57813800

$-2.29352100$

$-1.18712900$

$-1.16876200$

0.41666300 
2A (B3LYP/6-31G**)

3.06938910

3.47797300

2. 58626600

3.00050000

1. 86725400

0.76090700

$-0.58575700$

$-1.72229100$

$-3.08268810$

$-3.89855600$

$-3.06939410$

$-3.47797490$

$-2.58626700$

$-3.00050590$

$-1.86725900$

$-0.76090900$

0.58575500

1.72228710

3. 08268190

3.89855000

1. 76707800

$-1.76708300$

$-1.22273300$

1. 22273400

5.11061810

4.00591800

1.77829300

$-0.16563800$

$-3.38260790$

$-4.97456690$

$-5.11059380$

$-4.00592710$

$-1.77830100$

3.38260290

4.97455980

0.97144800

$-0.97145200$

0.85014400

0.57197000

0.81696300

1. 34283400

1. 62332700

1. 38017900

0.16573900

0.59948600

1. 53285210

2. 02904390

1. 59470000

$-0.85014200$

$-0.57190900$

$-0.81689300$

$-1.34281400$

$-1.62336400$

$-1.38022490$

$-0.59937000$

$-1.53282500$

$-2.02911900$

$-1.59479000$

$-4.96588900$

$-5.79834080$

$-7.44293500$

$-8.14620780$

$-7.42898320$

$-5.78612420$

$-8.03346440$

$-7.32986780$

$-8.27214150$

$-9.15048890$

$-8.01193620$

$-7.31175800$

4. 96588990

5.78625920

7.42911910

8.14620490

7.44280910

5. 79818680

8. 01214790

7. 31193300

8. 27211280

9.15049550

8.03325270

7. 32973910
$-0.70746900$

0.63903300

1. 73913500

3.13822200

3.87575510

2.93030810

3.35084610

2. 52651690

2. 97410490

1.87742400

0.70748200

$-0.63902200$

1.73912100

$-3.13820200$

$-3.87573600$

$-2.93029500$

$-3.35083290$

$-2.52650400$

$-2.97409390$

-1.87741300

$-1.15169800$

1.15171300

$-1.64582800$

1. 64584000

2. 00932690

3.52375700

4. 94901420

5.11498310

4.00811580

1. 86417500

$-2.00934100$

$-3.52372500$

$-4.94898990$

$-4.00810810$

$-1.86416600$

-0. 52404400

0.52406100

$-4.82916020$

$-5.60056590$

$-6.9741731$

$-7.59899380$

$-6.84200620$

$-5.46800610$

$-5.11505600$

$-7.55494210$

$-8.66831020$

$-7.32067820$

$-4.88142490$

4. 82917310

5. 60053110

6.97413920

7. 59900900

6.84206910

5.46806810

7. 55487010

8.66832730

7.32077980

4.88152600

$-0.93483400$

$-0.42679600$

$-1.18643610$

$-0.68568600$

$-1.06147100$

$-0.26798400$

$-0.94526200$

$-2.27738000$

0.40078600

$-1.13152600$

$-0.73781600$

$-2.14988990$

0.93482800

0.26783400

1. 06129900

0.68563400

1.18652400

0.42691400

0.73753000

2.14971300

$-0.40083500$

1.13145400

0.94545500

2. 27746390
0.02700900

0.01933000

.03135600

0.11960200

0.11869300

0.02235500

$-0.00556800$

$-0.02882200$

0.10791500

$-0.09993900$

$-0.02702700$

$-0.01929100$

$-0.03136200$

$-0.11967600$

$-0.11884100$

$-0.02248100$

0.00540200

0.02869200

0.10780000

0.09989600

$-0.01063500$

0.01057600

0.01815500

$-0.01821400$

$-0.05914100$

0.20106600

0.19124000

$-2.03282900$

$-0.17228100$

$-0.15517000$

0.05919200

$-0.20115900$

$-0.19146000$

0.17212800

0.15515300

$-0.06399100$

0.06393700

0.01181800

1.15038600

1.15751800

0.02548500

$-1.11316800$

$-1.11940000$

2. 03261090

2. 04931090

0.03063100

$-1.99981300$

$-2.00772400$

$-0.01205700$

$-1.15064300$

$-1.15784300$

$-0.02586000$

1. 11281100

1.11911000

$-2.04964900$

$-0.03105800$

1. 99941800

2. 00744890

$-0.00436200$

$-1.58224900$

$-1.26726900$

$-0.00253100$

1. 29736400

1. 52004800

$-2.15573310$

$-1.23809700$

$-0.05455200$

0.02421100

2.16438600

1.37206400

0.00450000

$-1.51977300$

$-1.29701300$

0.00299200

1. 26760700

1. 58251200

$-2.16394090$

$-1.37184500$

0.05513800

$-0.02369500$

2.15615610

1. 23830000 
2B (B3LYP/6-31G**)

3.03854400 3.47029600

2. 59989300

2. 93167500

1. 76827300

0.67228300

$-0.68879000$

$-1.78492500$

$-3.16462300$

$-3.94590800$

$-3.03854500$

$-3.47029700$

$-2.59989300$

$-2.93167600$

$-1.76827400$

$-0.67228300$

0.68878900

1. 78492400

3.16462300

3.94590800

1. 73483300

$-1.73483500$

$-1.22545500$

1. 22545500

5.08220100

3.92481300

1. 66410200

$-0.32922700$

$-3.47840100$

$-5.02150700$

$-5.08219800$

$-3.92481400$

$-1.66410400$

3.47840100

5.02150800

$-0.71912600$

0.71912700

0.98657300

0.73578100

1.01312700

1. 54312500

1. 79544000

1. 51996400

0.32922900

0.81860300

1. 75906800

2.20544700

1. 71540600

$-0.98657300$

$-0.73577900$

$-1.01312300$

$-1.54312100$

$-1.79543800$

$-1.51996300$

$-0.81859800$

$-1.75906200$

$-2.20544400$

$-1.71540600$

$-4.95355200$

$-5.79244000$

$-7.41851100$

$-8.13508500$

$-7.41125600$

$-5.78868100$

$-8.01367900$

$-7.28047600$

$-8.28525800$

$-9.12899900$

$-8.00357400$

$-7.26850300$

4.95355100

5.78876600

7.41133000

8.13508500

7.41844000

5.79235000

8.00369700

7.26858500

8.28525100

9.12900200

8.01355700

7.28040700
$-0.66513600$

0.68086400

1. 78903200

3.18321200

3.90186100

2.98247300

3.33000700

2.44308000

2. 91222000

1.80938300

0.66513700

$-0.68086300$

$-1.78903200$

3.18321100

$-3.90185900$

$-2.98247200$

$-3.33000600$

$-2.44308000$

$-2.91222100$

$-1.80938400$

$-1.07840400$

1.07840500

$-1.72305500$

1.72305500

2. 07837800

3. 59824000

4.97354000

5.12069700

3. 94265300

1. 76208400

$-2.07839400$

$-3.59823700$

$-4.97353700$

$-3.94265500$

$-1.76208700$

$-0.84430900$

0.84430800

$-4.80201400$

$-5.58923400$

$-6.95689400$

$-7.55970800$

$-6.78683100$

$-5.41915100$

$-5.12073600$

$-7.54961600$

$-8.62409200$

$-7.24834000$

$-4.81946400$

4.80201500

5. 58921300

6.95687400

7. 55971000

6.78685500

5.41917400

7. 54957800

8.62409400

7. 24838100

4. 81950400

$-1.00102100$

$-0.52120200$

$-1.31909700$

$-0.82827700$

$-1.18068600$

$-0.34600300$

$-1.09663200$

$-2.40714000$

0.25479000

$-1.29672100$

$-0.86712800$

$-2.26583600$

1. 00101900

0.34566200

1.18039000

0.82827100

1.31937400

0. 52155200

0.86663600

2. 26552300

$-0.25478400$

1. 29670500

1. 09711100

2. 40740900
0.03412500

0.01616800

0.01535200

0.08841600

0.08659100

0.00347600

$-0.02020400$

$-0.03984600$

$-0.12218200$

$-0.11258300$

$-0.03414700$

$-0.01617300$

$-0.01537300$

$-0.08844900$

$-0.08664400$

$-0.00352700$

0.02013900

0.03979000

0.12211000

0.11252000

$-0.00249500$

0.00246200

0.03100200

$-0.03103600$

$-0.06537800$

0.16083700

0.14942200

$-2.04057300$

$-0.19027500$

$-0.16743200$

0.06514500

$-0.16086700$

$-0.14948900$

0.19017700

0.16735400

0.08308800

$-0.08312100$

0.01510900

1.14910400

1.14280100

0.00099400

$-1.13374500$

$-1.12659700$

2. 04047400

2. 03203900

$-0.00432000$

$-2.02747800$

$-2.01043200$

$-0.01520200$

$-1.14921100$

$-1.14293500$

$-0.00114000$

1.13361400

1.12649200

$-2.03218400$

0.00415400

2. 02733800

2. 01033900

$-0.00314200$

$-1.58573300$

$-1.26849300$

$-0.00713000$

1. 29583500

1. 51727900

$-2.15875700$

$-1.23335500$

$-0.06490900$

0.02043200

2.16022000

1.37571400

0.00314300

-1. 51708400

$-1.29573500$

0.00735000

1. 26856200

1. 58588900

$-2.16001600$

$-1.37586500$

0.06538000

$-0.02026000$

2.15891000

1. 23317100 
2A (B3LYP/6-31G); no imaginary frequency

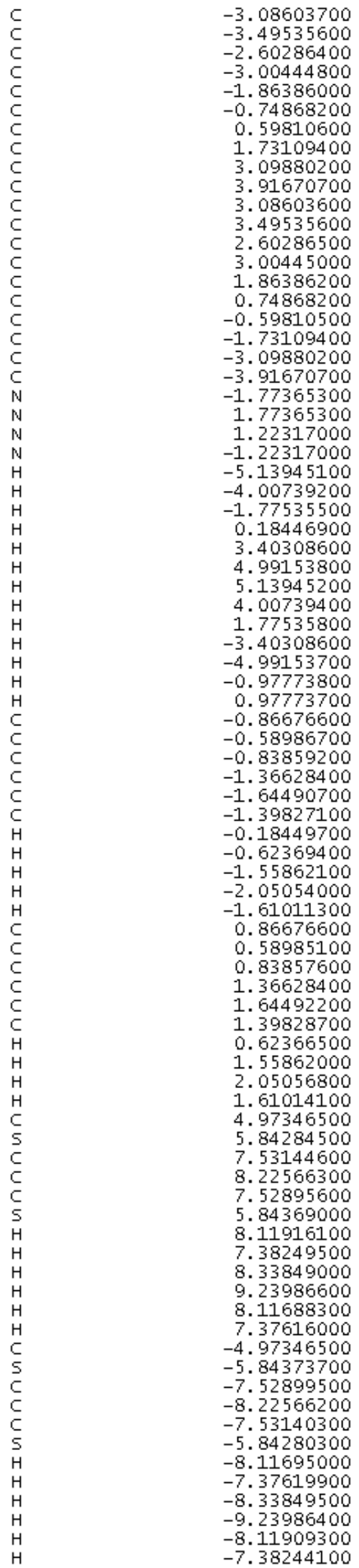

0.69927700 $-0.64719400$ $-1.74537500$ $-3.15300700$ $-3.89033900$ $-2.94670100$ $-3.36100200$ $-2.53078600$ $-2.97572300$ $-1.87417800$ $-0.69927300$ 0.64719800 1. 74537900 3.15300900 3.89034200 2.94670500 3.36100600 2. 53079100 2.97573000 1.87418500 1.14576100 $-1.14575700$ 1.64927400 $-1.64926900$ $-2.01872000$ $-3.54425300$ $-4.96279900$ $-5.13146200$ $-4.00733500$ $-1.86436700$ 2. 01872000 3. 54425300 4. 96280000 4. 00734400 1.86437600 0. 51632600 $-0.51632300$ 4. 84106900 5.61532000 6.99216700 7. 61677000 6.85643800 5.47917300 5.13151300 7. 57406300 8.68501600 7.33393900 4. 89206000 $-4.84106400$ $-5.61528900$ $-6.99213600$ $-7.61676600$ $-6.85646100$ $-5.47919500$ $-7.57401200$ $-8.68501100$ $-7.33398200$ $-4.89210300$ 0.94779400 0.38588000 1. 20146500 0.70933800 1.11761700 0.27608200 0.95592400 2. 28717300 $-0.38023900$ 1.13954100 0.81811400 2.20205600 $-0.94779300$ $-0.27607600$ $-1.11761400$ $-0.70935600$ $-1.20149600$ $-0.38589500$ $-0.81810000$ $-2.20205200$ 0.38022000 $-1.13956400$ $-0.95597400$ $-2.28720200$
0.01701600 0.01445700 0.02367800 0.09680700 0. 09718500 0.01673800 $-0.00265100$ $-0.01851900$ $-0.08175700$ $-0.07445800$ $-0.01701000$ $-0.01442000$ $-0.02367500$ $-0.09682600$ $-0.09724200$ $-0.01679100$ 0.00257500 0.01846300 0.08168400 0.07442800 $-0.01371500$ 0.01369800 0.01749300 $-0.01751000$ $-0.03297600$ 0.16318600 0.15979800 $-2.03303100$ $-0.13499900$ $-0.11766300$ 0.03308200 $-0.16320300$ $-0.15988300$ 0.13488900 0.11763000 $-0.04762100$ 0.04761400 0.00813700 1.15006800 1.15578800 0.01952100 $-1.12232400$ $-1.12783900$ 2. 03292000 2.04666500 0.02386800 $-2.00882500$ $-2.01574800$ $-0.00825100$ $-1.15019600$ $-1.15595200$ $-0.01970700$ 1.12215300 1.12770300 $-2.04683900$ $-0.02408100$ 2. 00863700 2. 01562300 $-0.00877200$ $-1.62190200$ $-1.29039400$ $-0.01703400$ 1. 28439800 1. 56071200 $-2.17859700$ $-1.26842000$ $-0.05229500$ $-0.00225300$ 2.15576300 1. 33135900 0.00886300 $-1.56059200$ $-1.28423800$ 0.01722100 1. 29055300 1. 62202200 $-2.15558100$ $-1.33122000$ 0.05250100 0.00246700 2.17877800 1. 26855900 
2C (B3LYP/6-31G**)

\begin{tabular}{|c|c|c|c|}
\hline \multirow{2}{*}{\multicolumn{4}{|c|}{-2.87898700}} \\
\hline & & 0.13193400 & -0.00365200 \\
\hline$c$ & -2.86712100 & 1.38212400 & -0.03998300 \\
\hline c & -3.54116400 & 2.67358900 & -0.15628600 \\
\hline$c$ & -2.56995100 & 3.61452500 & -0.17159100 \\
\hline$c$ & -1.30408500 & 2.90051700 & -0.05546400 \\
\hline$c$ & -0.06296300 & 3.56945600 & -0.02660900 \\
\hline$c$ & 1.20986200 & 2.97506700 & 0.01581900 \\
\hline c & 2.45623700 & 3.67325100 & 0.11590300 \\
\hline c & 3.46552300 & 2.74987300 & 0.12774000 \\
\hline C & 2.88096500 & 1.44288500 & 0.04259700 \\
\hline$c$ & 3.53091800 & 0.19103100 & 0.0354990 \\
\hline C & 2.85841700 & -1.05090600 & 0.0240180 \\
\hline C & 3.54012000 & -2.34409500 & 0.0718150 \\
\hline$c$ & 2.56971900 & -3.28439300 & 0.0611630 \\
\hline$c$ & 1. 29897900 & -2.56812100 & -0.0017300 \\
\hline$C$ & 0.05767700 & -3.23964900 & -0.0220960 \\
\hline$c$ & -1.21452000 & -2.64545200 & -0.0244080 \\
\hline$c$ & -2.46801100 & -3.34123800 & -0.0860930 \\
\hline C & -3.47471000 & -2.41757900 & -0.0664200 \\
\hline N & -1.51583700 & -1.30391700 & 0.0175460 \\
\hline N & 1. 51914500 & 1.63415600 & -0.0174950 \\
\hline N & 1. 50007700 & -1.21743200 & -0.0168110 \\
\hline N & -1.51187900 & 1. 55137500 & 0.0085060 \\
\hline $\mathrm{H}$ & -5.39254500 & 1.17175100 & 0.0920730 \\
\hline $\mathrm{H}$ & -4.60128800 & 2.85718300 & -0.2488010 \\
\hline $\mathrm{H}$ & -2.68849000 & 4.68305100 & -0.2680980 \\
\hline $\mathrm{H}$ & -0.87806600 & 5.24182200 & 1.9573440 \\
\hline $\mathrm{H}$ & 2.55380900 & 4.74534000 & 0.1824030 \\
\hline $\mathrm{H}$ & 4. 51761000 & 2.97290000 & 0.2116300 \\
\hline $\mathrm{H}$ & 5.37240500 & 1.26872000 & 0.0127840 \\
\hline $\mathrm{H}$ & 4.60630600 & -2.49614400 & 0.1197960 \\
\hline $\mathrm{H}$ & 2.68909400 & -4.35622200 & 0.1034170 \\
\hline $\mathrm{H}$ & -2.56895500 & -4.41335100 & -0.1467240 \\
\hline $\mathrm{H}$ & -4.53481500 & -2.60601400 & -0.1076740 \\
\hline $\mathrm{H}$ & -0.84799500 & -0.54127000 & 0.0621730 \\
\hline $\mathrm{H}$ & 0.85864500 & 0.86519500 & -0.0760980 \\
\hline$c$ & 0.07936200 & -4.74095800 & -0.0453430 \\
\hline$c$ & 0.49177700 & -5.43208100 & -1.1946820 \\
\hline C & 0.51119200 & -6.82689400 & -1.2185150 \\
\hline C & 0.11875800 & -7.55361800 & -0.0931560 \\
\hline c & -0.29340000 & -6.87710600 & 1.0559990 \\
\hline C & -0.31371500 & -5.48207100 & 1.0792490 \\
\hline $\mathrm{H}$ & 0.79472200 & -4.86805300 & -2.0716690 \\
\hline $\mathrm{H}$ & 0.83030900 & -7.34514700 & -2.1181970 \\
\hline $\mathrm{H}$ & 0.13453500 & -8.63943100 & -0.1114830 \\
\hline $\mathrm{H}$ & -0.59602700 & -7.43439500 & 1.9378900 \\
\hline $\mathrm{H}$ & -0.62911500 & -4.95735800 & 1.9761390 \\
\hline$c$ & -0.08287300 & 5.07056700 & -0.0356220 \\
\hline$c$ & -0.54040000 & 5.78685200 & 1.0810610 \\
\hline$c$ & -0.55870500 & 7.18188800 & 1.0742690 \\
\hline$c$ & -0.12094000 & 7.88417300 & -0.0499100 \\
\hline c & 0.33599900 & 7.18291400 & -1.1669300 \\
\hline$c$ & 0.35618700 & 5.78773400 & -1.1590700 \\
\hline $\mathrm{H}$ & -0.91228200 & 7.71955800 & 1.9493460 \\
\hline $\mathrm{H}$ & -0.13622500 & 8.97012100 & -0.0555620 \\
\hline $\mathrm{H}$ & 0.67358200 & 7.72104900 & -2.0480280 \\
\hline $\mathrm{H}$ & 0.70616000 & 5.24370000 & -2.0312790 \\
\hline$c$ & 5.04781300 & 0.23187100 & 0.0446290 \\
\hline 5 & 5.77034700 & -0.52056200 & -1.4883290 \\
\hline$c$ & 7.51613400 & -0.00627800 & -1.2275500 \\
\hline$c$ & 8.14469400 & -0.52095700 & 0.0708430 \\
\hline$c$ & 7.51124300 & 0.05633500 & 1.3401200 \\
\hline 5 & 5.76246000 & -0.43937100 & 1.617846 \\
\hline $\mathrm{H}$ & 8.05268200 & -0.40030000 & -2.0954050 \\
\hline $\mathrm{H}$ & 7.57724000 & 1.08824400 & -1.277313 \\
\hline $\mathrm{H}$ & 8.09587900 & -1.61453800 & 0.097312 \\
\hline $\mathrm{H}$ & 9.20742100 & -0.24031100 & 0.066347 \\
\hline $\mathrm{H}$ & 8.04263700 & -0.29689100 & 2.228448 \\
\hline $\mathrm{H}$ & 7.57541000 & 1.15178900 & 1.3375240 \\
\hline$c$ & -5.05112100 & 0.14290300 & 0.025792 \\
\hline 5 & -5.71929000 & -0.66298100 & 1. 5571750 \\
\hline$c$ & -7.48295100 & -0.18794100 & 1.3492590 \\
\hline C & -8.13054200 & -0.69271100 & 0.0564330 \\
\hline$C$ & -7.54327900 & -0.07440100 & -1.215555 \\
\hline 5 & -5.78833900 & -0.51393600 & -1.5436050 \\
\hline $\mathrm{H}$ & -7.98795200 & -0.61185300 & 2.221921 \\
\hline $\mathrm{H}$ & -7.56819100 & 0.90349900 & 1.4225560 \\
\hline $\mathrm{H}$ & -8.05546600 & -1.78404100 & 0.0066350 \\
\hline $\mathrm{H}$ & -9.19942400 & -0.43909400 & 0.092283 \\
\hline $\mathrm{H}$ & 08545300 & -0.42530500 & 98267 \\
\hline $\mathrm{H}$ & & & \\
\hline
\end{tabular}


2TS (B3LYP/6-31G); one imaginary frequency

\begin{tabular}{|c|c|}
\hline 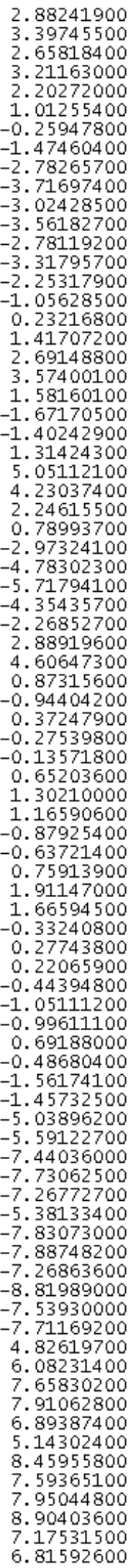 & 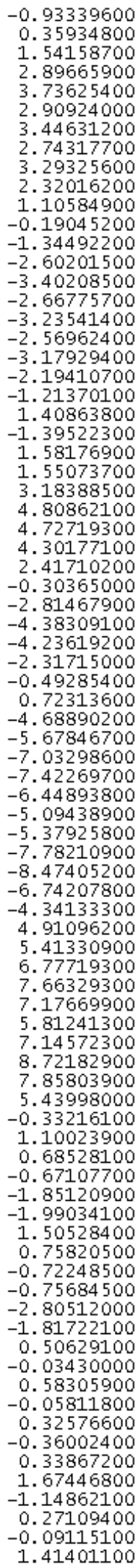 \\
\hline
\end{tabular}

0.20457600

0.41968100

.18256700

$-0.17469700$

$-0.37023100$

$-0.64395900$

$-0.88163900$

$-0.62991400$

$-0.27149100$

$-0.09806000$

$-0.38686900$

$-0.92408500$

$-1.19393900$

$-0.78736400$

$-0.76440300$

$-0.39217800$

$-0.11901300$

0.25623500

$-0.17971900$

$-0.31778100$

$-0.35780700$

$-0.14966700$

1. 06631600

0.39057500

$-0.27680800$

$-2.82075600$

$-1.20810300$

$-0.72513300$

$-0.56687700$

$-1.11886200$

$-1.64066200$

$-0.18148700$

0.53362000

$-0.28053700$

$-0.14186600$

$-1.10540500$

$-0.34072400$

$-0.65989200$

$-1.74950400$

$-2.51693400$

$-2.19632800$

0.50937500

$-0.05514400$

$-1.99729200$

$-3.36615400$

$-2.79674200$

$-0.98878900$

$-2.15426700$

$-2.45946700$

$-1.60333400$

$-0.43971100$

$-0.13482300$

$-3.36533700$

$-1.83965600$

0.23373500

0.77411200

0.29288400

1.46458400

1. 61279000

2.25321200

1. 40052200

1. 20776400

2. 22134600

0.61471500

3.24532900

2. 39424000

1. 86032700

0.39890100

0.87454100

$-0.46801700$

0.40418100

1. 77222100

2. 85174000

2. 55241900

$-0.29789600$

0.48244200

1. 67011100

2.11686600

82211700

2. 95714600 
3A (B3LYP/6-31G**)

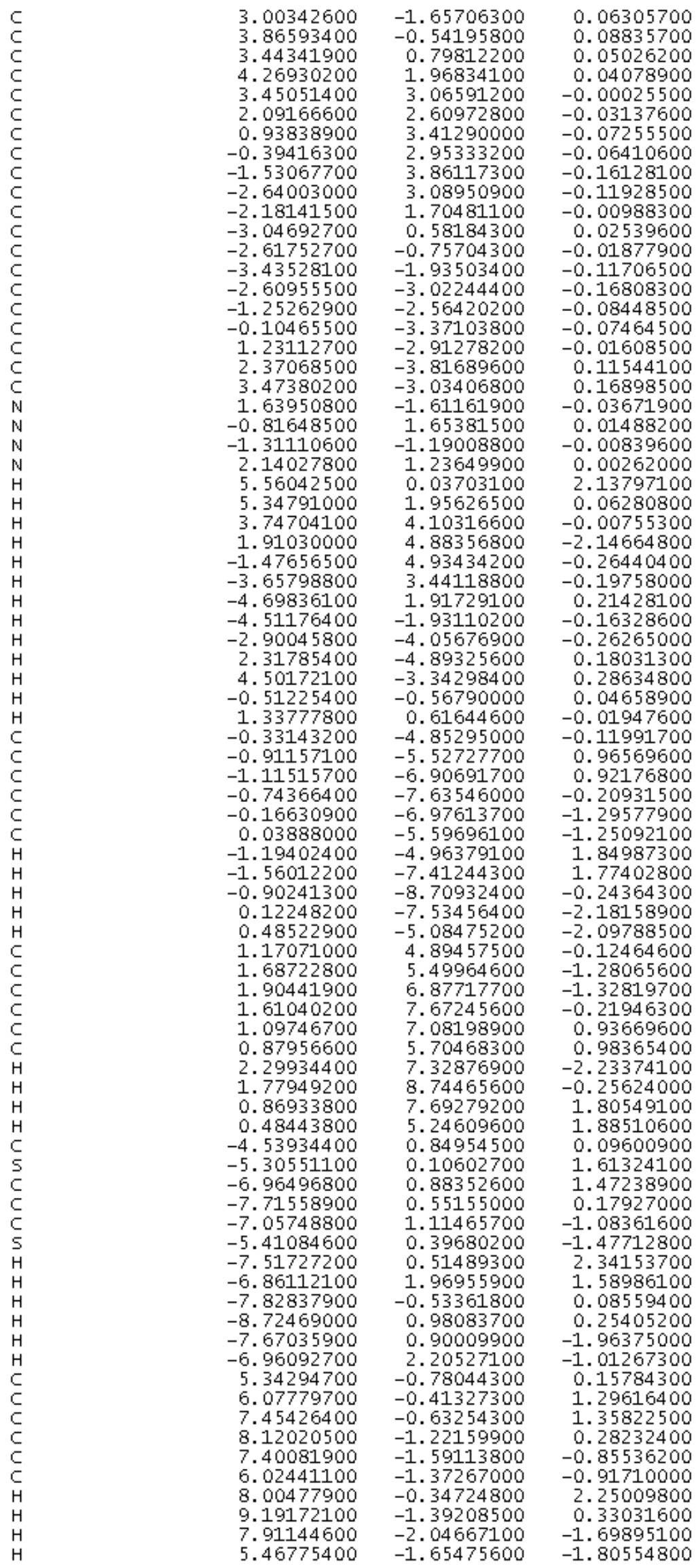


3B (B3LYP/6-31G**)

\begin{tabular}{|c|c|c|c|}
\hline \multirow{2}{*}{\multicolumn{4}{|c|}{-2.97687900}} \\
\hline & & & \\
\hline C & -3.84459900 & 0.68217600 & 0.06946800 \\
\hline$C$ & -3.43107400 & -0.66667600 & 0.03561500 \\
\hline 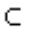 & -4.36294300 & -1.78902200 & 0.01665800 \\
\hline$c$ & -3.60552100 & -2.91064900 & -0.02162700 \\
\hline & -2.21507000 & -2.46632600 & -0.03746900 \\
\hline 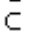 & -1.12490900 & -3.36274900 & -0.06276300 \\
\hline & 0.23389800 & -3.01122300 & -0.04043400 \\
\hline 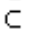 & 1.33890100 & -3.92011400 & -0.11094100 \\
\hline$c$ & 2.49562100 & -3.19231500 & -0.06859100 \\
\hline $\mathrm{C}$ & 2.15202400 & -1.80044400 & 0.0151830 \\
\hline$c$ & 3.02135600 & -0.69057800 & 0.03045600 \\
\hline 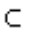 & 2.60003400 & 0.65806100 & -0.03633700 \\
\hline C & 3.52312800 & 1.78746800 & -0.15435200 \\
\hline 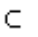 & 2.75738900 & 2.89945200 & -0.21061100 \\
\hline c & 1.37132100 & 2.45355600 & -0.11025400 \\
\hline & 0.28695700 & 3.35470000 & -0.10288400 \\
\hline & -1.07488000 & 3.00707600 & -0.05049100 \\
\hline & -2.18157300 & 3.91009100 & 0.07909500 \\
\hline $\mathrm{c}$ & -3.33268400 & 3.17038200 & 0.13372600 \\
\hline $\mathrm{N}$ & -1.60557900 & 1.73985200 & -0.07435300 \\
\hline $\mathbb{N}$ & 0.77670400 & -1.74663800 & 0.0290180 \\
\hline N & 1.30262500 & 1.08955400 & -0.02257600 \\
\hline N & -2.13631400 & -1.10422100 & -0.0025620 \\
\hline $\mathrm{H}$ & -5.53161700 & 0.15862900 & 2.13550800 \\
\hline $\mathrm{H}$ & -5.44015300 & -1.71642400 & 0.02594100 \\
\hline H & -3.94112300 & -3.93671300 & -0.03540800 \\
\hline $\mathrm{H}$ & -2.12689900 & -4.77880900 & -2.15688500 \\
\hline $\mathrm{H}$ & 1.24553100 & -4.99123600 & -0.19796600 \\
\hline $\mathrm{H}$ & 3.49339300 & -3.59771800 & -0.13040400 \\
\hline $\mathrm{H}$ & 4.62022800 & -2.08769200 & 0.24304700 \\
\hline H & 4.59792800 & 1.72352600 & -0.20731700 \\
\hline H & 3.08569600 & 3.92170700 & -0.3209450 \\
\hline $\mathrm{H}$ & -2.08938800 & 4.98305800 & 0.14259100 \\
\hline $\mathrm{H}$ & -4.34131100 & 3.53583100 & 0.2489260 \\
\hline $\mathrm{H}$ & -1.07175200 & 0.87915000 & -0.13227300 \\
\hline $\mathrm{H}$ & 0.25033000 & -0.88090000 & 0.0852940 \\
\hline$c$ & 0.58848000 & 4.82367700 & -0.12768900 \\
\hline $\bar{c}$ & 1.19831900 & 5.44961200 & 0.97071700 \\
\hline $\bar{c}$ & 1.47120300 & 6.81750900 & 0.95054300 \\
\hline$c$ & 1.13968800 & 7.58332000 & -0.16850100 \\
\hline $\bar{c}$ & 0.53280200 & 6.97274300 & -1.26707700 \\
\hline $\bar{c}$ & 0.25808000 & 5.60489100 & -1.24589000 \\
\hline $\mathrm{H}$ & 1.45280800 & 4.85648200 & 1.84376700 \\
\hline $\mathrm{H}$ & 1.94015900 & 7.28511100 & 1.81153400 \\
\hline $\mathrm{H}$ & 1.35323400 & 8.64808300 & -0.1843480 \\
\hline $\mathrm{H}$ & 0.27601700 & 7.55972800 & -2.14425300 \\
\hline $\mathrm{H}$ & -0.20851900 & 5.13062900 & -2.10414500 \\
\hline$c$ & -1.42739600 & -4.83149100 & -0.12163300 \\
\hline $\bar{c}$ & -1.94792700 & -5.40816500 & -1.29033400 \\
\hline C & -2.22959700 & -6.77342200 & -1.34692000 \\
\hline $\bar{c}$ & -1.99553900 & -7.58516100 & -0.23564700 \\
\hline C & -1.47791800 & -7.02328000 & 0.93240100 \\
\hline 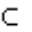 & -1.19560500 & -5.65783000 & 0.98852400 \\
\hline $\mathrm{H}$ & -2.62889700 & -7.20293500 & -2.26123700 \\
\hline $\mathrm{H}$ & -2.21553500 & -8.64780900 & -0.27959100 \\
\hline $\mathrm{H}$ & -1.29720000 & -7.64646300 & 1.80356100 \\
\hline $\mathrm{H}$ & -0.79953500 & -5.22129800 & 1.90058900 \\
\hline$c$ & 4.50249600 & -1.01591400 & 0.10502000 \\
\hline 5 & 5.29951900 & -0.28072800 & 1.60915000 \\
\hline$c$ & 6.92059600 & -1.13794100 & 1.48326500 \\
\hline C & 7.68566400 & -0.86278500 & 0.18518600 \\
\hline $\bar{c}$ & 7.00369800 & -1.41877500 & -1.06829700 \\
\hline 5 & 5.39114400 & -0.63434600 & -1.47689600 \\
\hline $\mathrm{H}$ & 7.48962500 & -0.78079900 & 2.34639700 \\
\hline $\mathrm{H}$ & 6.76668900 & -2.21604600 & 1.61876800 \\
\hline $\mathrm{H}$ & 7.84595900 & 0.21452700 & 0.07241500 \\
\hline $\mathrm{H}$ & 8.67489600 & -1.33470500 & 0.26846200 \\
\hline $\mathrm{H}$ & 7.62595200 & -1.24725000 & -1.95129800 \\
\hline $\mathrm{H}$ & 6.85999100 & -2.50287400 & -0.97786600 \\
\hline$c$ & -5.30965800 & 0.97962000 & 0.1577500 \\
\hline$c$ & -6.04189700 & 0.63858100 & 1.3060310 \\
\hline 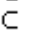 & -7.40610700 & 0.91704000 & 1.39078000 \\
\hline 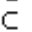 & -8.06270700 & 1. 54144900 & 0.3289210 \\
\hline$c$ & -7.34607100 & 1.88591800 & -0.81813200 \\
\hline C & -5.98136000 & 1.60835400 & -0.90250100 \\
\hline $\mathrm{H}$ & -7.95474900 & 0.64947000 & 2.28925900 \\
\hline $\mathrm{H}$ & -9.12496600 & 1.75773300 & 0.3948290 \\
\hline $\mathrm{H}$ & -7.84969500 & 2.36711400 & -1.65163700 \\
\hline & -5.42821300 & 1.86898000 & -1.799810 \\
\hline
\end{tabular}


3A (B3LYP/6-31G); no imaginary frequency

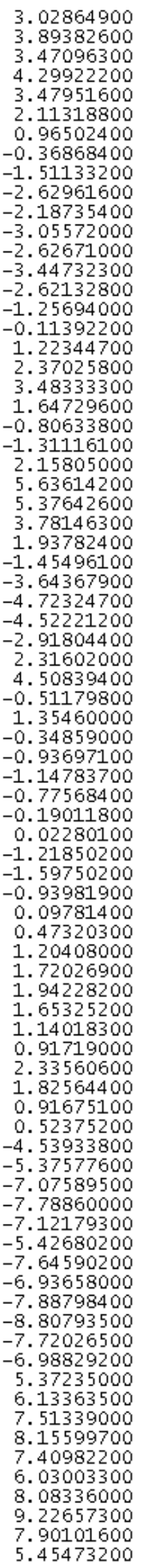

3. 02864900

3.47096300

0.96502400

..36868400

$-2.62961600$

$-3.05572000$

$-2.62671000$

$-3.44732300$

$-0.11392200$

1. 22344700

3. 48333300

1. 64729600

80633800

2.15805000

.63614200

3.78146300

1.93782400

$-3.64367900$

$-4.72324700$

52221200

2. 31602000

4. 50839400

- 1.35460000

$-0.34859000$

$-1.14783700$

$-0.77568400$

$-0.19011800$

$-1.21850200$

$-1.59750200$

0.09781400

20408000

1. 72026900

1.65325200

14018300

0.91719000

3560600

0.91675100

200

$-5.37577600$

$-7.78860000$

$-7.12179300$

$-7.64590200$

$-6.93658000$

$-7.88798400$

$-7.80793500$

$-6.98829200$

6.13363500

7. 51339000

8.15599700

40982200

8.08336000

5.45473200
$-1.66685200$

$-0.55549500$

0.78493700

1.95926400

3.06292200

2.61214600

3.42406200

2. 97368100

3.88323800

3.11337900

1. 71706300

0.59738800

$-0.74255700$

$-1.92534200$

$-3.01965500$

$-2.56528500$

$-3.37990400$

$-2.93034000$

$-3.83353500$

$-3.05333600$

$-1.61744600$

1. 66067100

$-1.17978500$

1. 22853400

0.06001600

1.94840200

4.09691000

4. 92286700

4.95703100

3.47569000

1.93669200

$-1.92409900$

$-4.05225900$

$-4.90765900$

$-3.37045500$

$-0.55616100$

0.60874000

$-4.86341500$

$-5.51874900$

$-6.90163900$

$-7.65182600$

$-7.01098900$

$-5.62859400$

$-4.94047600$

$-7.39165500$

$-8.72462500$

$-7.58491800$

$-5.13240800$

4.90773600

5. 52756700

6.90870800

7.69219000

7.08647900

5.70559100

7.37140800

8.76359300

7.68651700

5.23662400

0.87075600

0.14631100

0.97193100

0.58692500

1.11972800

0.33792500

0.64325700

2.05688600

-0. 50286500

1. 00306400

0.88727800

2.20668700

$-0.80042300$

$-0.41081600$

$-0.63835200$

$-1.25794900$

$-1.65033900$

$-1.42424400$

$-0.33715000$

$-1.43408600$

$-2.12819600$

$-1.72258300$
0.04916200

0.05922500

0.02798000

0.01154800

$-0.01076900$

$-0.02518700$

$-0.04684500$

$-0.03722300$

$-0.11149600$

$-0.08572600$

$-0.00605900$

0.01188200

$-0.01525400$

$-0.09181300$

$-0.12384300$

$-0.05329000$

$-0.03879100$

0.00234300

0.12615900

0.15759600

$-0.03237400$

0.01787700

0.00119200

$-0.00030000$

2. 06583500

0.01579300

$-0.01675500$

$-2.10775900$

$-0.18856100$

$-0.14596100$

0.12276800

$-0.13271800$

$-0.19767300$

0.20124800

0.26421100

0.03627100

$-0.01021100$

$-0.06235000$

1. 03568100

1. 01253000

$-0.10914000$

$-1.20738300$

$-1.18390800$

1. 90985000

1. 87051700

$-0.12705000$

$-2.08269300$

$-2.03748800$

$-0.07981700$

$-1.23309700$

$-1.26261700$

$-0.13916400$

1. 01390300

1.04349700

$-2.16244800$

$-0.16215800$

1.89056200

1. 93965600

0.04839000

1. 61306300

1. 38452100

0.08452000

$-1.18720100$

$-1.56516500$

2. 25730600

1.45609500

0.02384700

0.12641700

$-2.07175200$

$-1.14079200$

0.10547300

1. 22401900

1. 26364300

0.18546600

$-0.93208000$

$-0.97190200$

2.13707500

0.21619100

$-1.77400200$

$-1.84226200$ 
3TS (B3LYP/6-31G); one imaginary frequency

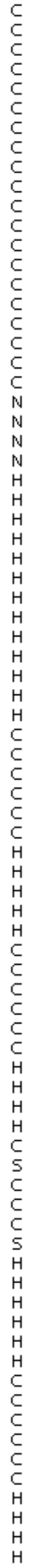

\begin{tabular}{|c|c|}
\hline $\begin{array}{r}-3.07463600 \\
-3.77725000 \\
-3.23326900 \\
-4.00809200 \\
-3.16038900 \\
-1.85453200 \\
-0.69312500 \\
0.61213700 \\
1.81264500 \\
2.89065900 \\
2.40339700 \\
3.13913200 \\
2.53648100 \\
3.24436800 \\
2.31425900 \\
1.03509200 \\
-0.13805100 \\
-1.39848300 \\
-2.54019100 \\
-3.54839900 \\
-1 \\
76667000\end{array}$ & 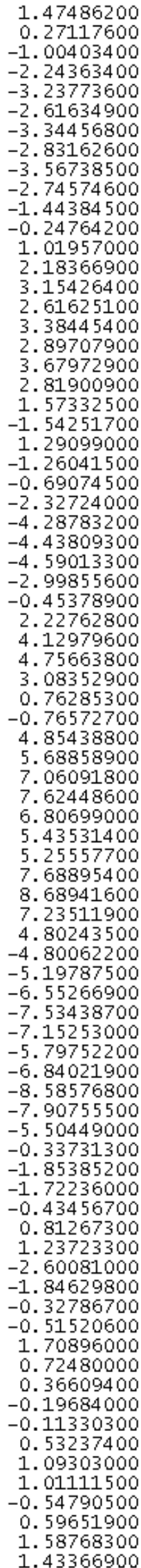 \\
\hline
\end{tabular}

0.39767600

0. 59630700

0.34570900

0.35207600

$-0.03048000$

$-0.25805700$

$-0.60524500$

$-0.63222600$

$-0.93037000$

$-0.72103200$

$-0.32818100$

$-0.17181100$

$-0.41869700$

$-0.96835000$

$-1.16659800$

$-0.70751300$

$-0.59968900$

$-0.19183100$

0.19641000

0.57081700

$-0.04828300$

$-0.31347700$

$-0.31869700$

$-0.02388500$

2. 90960600

0.59657800

$-0.13613100$

$-2.71603200$

$-1.26737600$

$-0.86746900$

$-0.72365000$

$-1.21687400$

$-1.59920000$

0.21503600

0.93816400

$-0.21058100$

$-0.09467600$

$-0.87871300$

$-0.13268700$

$-0.39399600$

$-1.40631000$

$-2.15429000$

$-1.89085000$

0.65812100

0.19569200

$-1.60960400$

$-2.94450200$

$-2.47713600$

$-0.92856500$

$-2.06966500$

$-2.37698900$

$-1.54713500$

$-0.40770000$

$-0.10094100$

$-3.26397700$

$-1.78499900$

0.24531400

0.78906400

0.16117500

1. 29843300

1. 39251900

2. 03738100

1. 20955700

1.07964300

1. 97981600

0.38036900

3.04287000

2.14726200

1. 66772700

0.19400300

1.06798300

2. 30318800

2.75408000

1. 97800800

0.74700100

0.29563900

3.71151900

2.32795500

0.13478600

$-0.66615200$ 
4 (B3LYP/6-31G**)

$C$
$C$
$C$
$C$
$C$
$C$
$C$
$C$
$C$
$C$
$C$
$C$
$C$
$C$
$C$
$C$
$C$
$C$
$C$
$C$
$C$
$N$
$N$
$H$
$H$
$H$
$H$
$H$
$H$
$H$
$H$
$H$
$H$
$H$
$H$
$H$
$H$
$H$
$H$
$H$
$H$
$H$
$-0.64126000$

0.63004500

1. 76374300

3.09990500

3.90694200

3.06792500

3. 55048300

2. 74097200

3.23244600

2. 21519400

1.06677000

$-0.22973700$

$-1.33977300$

$-2.66502500$

$-3.40394600$

$-2.56928400$

$-3.03957900$

$-2.26334000$

$-2.78691000$

$-1.78934700$

$-0.94563200$

1. 39816500

$-1.29249800$

1. 75488600

0.75500500

3.36546100

4. 97508700

4. 72741300

4.23303100

2. 22540300

0.58031700

$-2.96025700$

$-4.43158100$

$-3.79730300$

$-1.80428800$

0.22835800

$-4.43224800$

$-5.55216900$

$-6.84322400$

$-7.03666100$

$-5.93088200$

$-4.63971700$

$-5.40477400$

$-7.69787200$

$-8.04196300$

$-6.07184500$

$-3.78042800$

4.98706100

5.43725800

6.77630900

7. 68932300

7.25556200

5.91622100

7.10500100

8.73199400

7. 96123000

5. 58415700

$-0.38831300$

$-0.87445500$

$-0.71420500$

$-1.58915400$

$-1.21193100$

$-1.49518400$

$-0.98967900$

0.34118300

$-2.64196900$

$-1.47853800$

$-1.82263000$

$-0.16312600$
$-3.70044900$

$-4.06564900$

$-3.36722200$

$-3.88383800$

$-3.01725900$

$-1.93828900$

$-0.75910300$

0.37336100

1. 69320800

2. 55759200

1. 76378100

2.27564800

1. 43768500

1.89565300

0.79053400

$-0.35128600$

$-1.66288800$

$-2.67232400$

$-3.94237300$

$-4.56104400$

$-2.53785100$

0.42665100

0.05858000

$-2.16616700$

$-5.00784400$

$-4.80796000$

$-3.07109300$

$-2.05321000$

1. 92216200

3.62878000

4. 18649600

2. 93005000

0.74268600

$-4.28263900$

$-5.52534500$

$-1.05403200$

$-1.98928900$

$-1.51367100$

$-1.84139600$

$-2.65249800$

$-3.13717400$

$-2.81024000$

$-0.89319300$

$-1.46707400$

$-2.90701600$

$-3.76874200$

$-3.18548500$

$-0.69448100$

$-1.44411200$

$-1.40465300$

$-0.61924500$

0.12443900

0.08637200

$-1.98645400$

$-0.58914000$

0.73073300

0.65493900

3. 77125000

4. 62891800

6.36701600

6. 72217900

5.97368200

4.15894600

6.97376300

6. 57223000

6. 53937900

7.79884900

6.31872500

6.16147900
$-1.35525500$

$-1.75857700$

$-1.38108100$

$-1.48504500$

$-0.81628800$

$-0.35674600$

0.21625700

0.30840900

0.60180300

0.35276300

$-0.00610500$

$-0.14035100$

$-0.00516800$

0.33585200

0.60707900

0.33629000

0.27113700

$-0.30436800$

$-0.74472200$

$-1.43064900$

$-0.67974400$

$-0.02179000$

$-0.00253700$

$-0.70576300$

$-2.28087300$

$-1.98072600$

$-0.66908100$

2. 27392700

0.93485100

0.48730200

$-0.59303700$

0.41145100

0.93268600

$-0.57502400$

$-1.92071800$

$-0.35791900$

0. 70634800

0.00634100

0.42129200

1. 54067900

2.24077400

1. 82567900

$-0.87231700$

$-0.13489200$

1. 86336500

3.11317800

2. 37343300

0.62444800

1. 72236800

2.11174500

1. 40640400

0.30772300

$-0.08092900$

2. 96806200

1. 70881100

$-0.25295200$

$-0.94419900$

$-0.31511100$

1. 25682300

0.67725100

$-0.52804700$

$-1.80984800$

$-1.74686100$

1. 54457500

0.45862100

$-0.28900800$

$-0.71945400$

$-2.64908500$

$-2.07181800$ 
4 (B3LYP/6-31G); no imaginary frequency

\begin{tabular}{|c|c|c|c|}
\hline $\begin{array}{l}C \\
C \\
C \\
C \\
C \\
C \\
C \\
C \\
C \\
C \\
C \\
C \\
C \\
C \\
C \\
C \\
C \\
C \\
\mathrm{C} \\
\mathrm{C} \\
\mathrm{N} \\
\mathrm{C} \\
\mathrm{C} \\
\mathrm{C} \\
\mathrm{C} \\
\mathrm{C} \\
\mathrm{C} \\
\mathrm{H} \\
\mathrm{H} \\
\mathrm{H} \\
\mathrm{H} \\
\mathrm{H} \\
\mathrm{C} \\
\mathrm{C} \\
\mathrm{C} \\
\mathrm{C} \\
\mathrm{H} \\
\mathrm{H} \\
\mathrm{H} \\
\mathrm{H} \\
\mathrm{H} \\
\mathrm{H} \\
\mathrm{H} \\
\mathrm{N} \\
\mathrm{C} \\
\mathrm{C} \\
\mathrm{C} \\
\mathrm{C} \\
\mathrm{C} \\
\mathrm{C} \\
\mathrm{C} \\
\mathrm{C} \\
\mathrm{C} \\
\mathrm{C}\end{array}$ & 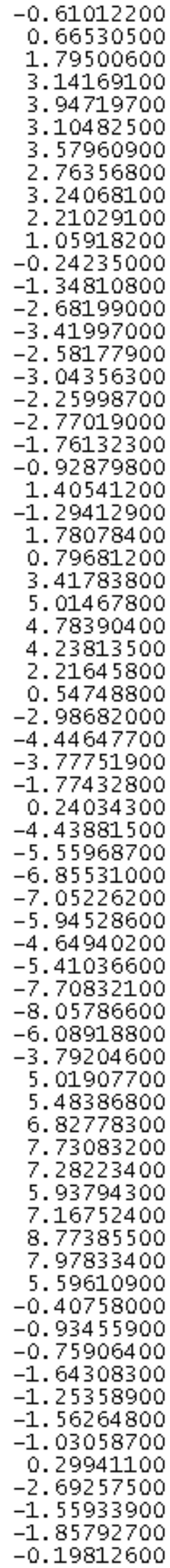 & 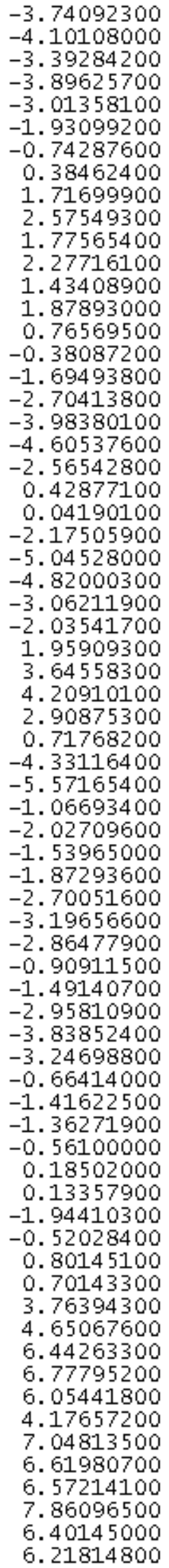 & 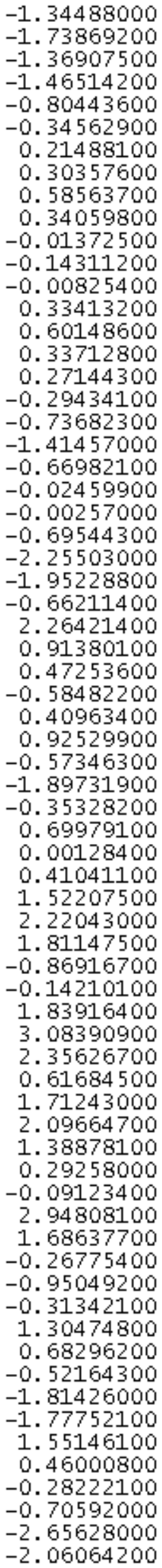 \\
\hline
\end{tabular}


4TS (B3LYP/6-31G); one imaginary frequency

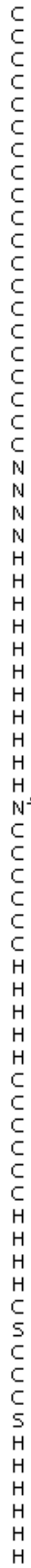

1. 21644600

0.00022500

$-1.21607700$

$-2.48155400$

$-3.37716500$

$-2.68042800$

$-3.28124300$

$-2.63404200$

$-3.26537300$

$-2.41856100$

$-1.20665600$

$-0.00010400$

1. 20658200

2. 41860500

3.26558800

2. 63421800

3.28143300

2. 68063100

3.37741400

2. 48191900

1.35752700

$-1.34012900$

1.34018100

$-1.35727100$

0.00027900

$-2.64545300$

$-4.41920800$

$-4.13373900$

$-4.23852700$

$-2.57699500$

0.00035300

2. 57702000

4. 23885900

4.41942200

2.64589200

0.00007600

4. 67994100

5.75125600

7.05363300

7.30904500

6.25380000

4. 95164400

5. 56109500

7.86803000

8.31993500

6.44265200

4.13396700

$-4.67975400$

$-4.95142900$

$-6.25358600$

$-7.30885000$

$-7.05344700$

$-5.75106900$

$-6.44242800$

$-8.31973500$

$-7.86784800$

$-5.56089800$

$-0.00012000$

1. 55774800

1.27627300

$-0.00049500$

$-1.27695300$

$-1.55869200$

2.16728300

1. 29483400

$-0.00081400$

$-0.00040600$

$-2.16815800$

$-1.29505500$
$-3.17187200$

$-3.60056100$

$-3.17181000$

$-3.83047400$

$-3.26345800$

$-2.21031300$

$-1.27916900$

$-0.08513600$

1. 06225700

2.12041300

1. 62162900

2.33729000

1. 62180500

2.12092500

1.06292900

$-0.08470500$

$-1.27880300$

$-2.21020600$

$-3.26354200$

$-3.83064600$

$-2.16224200$

0.25690200

0.25704800

$-2.16230500$

$-4.40420800$

$-4.64127900$

$-3.50638800$

$-3.28472100$

1. 06224700

3.13363000

4.36044200

3.13426900

1. 06316800

$-3.50650500$

$-4.64156700$

$-0.94692800$

$-1.53327900$

$-0.70680400$

$-0.97287200$

$-2.06999800$

$-2.90346600$

$-2.63936700$

0.13386600

$-0.32828200$

$-2.27523300$

$-3.75664500$

$-3.28403800$

$-1.53381900$

$-2.64001900$

$-2.90422800$

$-2.07072600$

$-0.97340900$

$-0.70723400$

$-3.75752100$

$-2.27603600$

$-0.32874800$

0.13362100

3.86246500

4. 49500100

6.36769300

6.87483500

6.36787200

4. 49515900

6.79522100

6.62212900

6. 59987500

7.97521200

6.79527000

6.62264200
1. 91042400

2. 41807600

1. 91067300

2.11920200

1. 25574800

0.54910000

$-0.30806100$

$-0.63281300$

$-1.23968800$

$-1.08614700$

$-0.46542400$

$-0.34800000$

$-0.46502100$

$-1.08527000$

$-1.23890500$

$-0.63253200$

$-0.30812400$

0.54877900

1. 25510800

2. 11862100

0.96405100

$-0.23208200$

$-0.23198400$

0.96418000

3.14591300

2. 81397200

1.12343500

$-1.90531100$

$-1.70249400$

$-1.41033600$

$-1.22448500$

$-1.40907300$

$-1.70147900$

1.12258700

2. 81323900

0.38902500

$-0.77115500$

$-0.38063200$

$-0.81637500$

$-1.64729900$

$-2.03614700$

$-1.59978700$

0.27835700

$-0.50047300$

$-1.98512400$

$-2.68025600$

$-1.90570000$

$-0.77101300$

$-1.59950000$

$-2.03579400$

$-1.64707000$

$-0.81639800$

$-0.38071700$

$-2.67975600$

$-1.98486200$

$-0.50065000$

0.27803100

$-0.24833300$

0.68902700

0.52217400

1.19161300

0. 52144800

0.68769100

0.98950500

$-0.54378500$

2.25219300

1.13854800

0.98853300

$-0.54443800$ 